\title{
Hepatic Ischemia and Reperfusion Injury and Trauma: Current Concepts
}

\author{
Dimitrios Papadopoulos ${ }^{1}$, Thomas Siempis ${ }^{1, *}$, Eleni Theodorakou ${ }^{1}$, Georgios Tsoulfas ${ }^{1}$ \\ ${ }^{1}$ 1st Department of Surgery, Aristotle University of Thessaloniki, Papageorgiou General Hospital, Thessaloniki, Greece \\ ${ }^{*}$ Corresponding author: Thomas Siempis, 1st Department of Surgery, Aristotle University of Thessaloniki, Papageorgiou General Hospital, Thessaloniki, Greece. Tel: +30-6978659716, \\ E-mail: thomassiempis@yahoo.com.
}

Received: May 26, 2013; Accepted: Jun 11, 2013

\begin{abstract}
Context: Ischemia-reperfusion injury is a fascinating topic which has drawn a lot of interest in the last several years. Hepatic ischemia reperfusion injury may occur in a variety of clinical situations. These include transplantation, liver resection, trauma, and vascular surgery. Evidence Acquisition: The purpose of this review was to outline the molecular mechanisms underlying hepatic $\mathrm{I} / \mathrm{R}$ injury and present the latest approaches, both surgical and pharmacological, regarding the prevention of it. A comprehensive electronic literature search in MEDLINE/PubMed was performed to identify relative articles published within the last 2 years.

Results: The basic mechanism of hepatic ischemia - reperfusion injury is one of blood deprivation during ischemia, followed by the return of flow during reperfusion. It involves a complex series of events, such as mitochondrial deenergization, adenosine-5'-triphosphate depletion, alterations of electrolyte homeostasis, as well as Kupffer cell activation, oxidative stress changes and upregulation of proinflammatory cytokine signaling. The great number of variable pathways, with several mediators interacting with each other, leads to a high number of candidates for potential therapeutic intervention. As far as surgical approaches are concerned, the modification of existing clamping techniques and the ischemic preconditioning are the most promising techniques till recently. In the search for novel techniques of protecting against hepatic ischemia reperfusion injury, many different strategies have been used in experimental models. The biggest part of this research lies around antioxidant therapy, but other potential solutions have been explored as well.

Conclusions: The management of hepatic trauma, in spite of the fact that it has become increasingly nonoperative, there still remains the possibility of hepatic resection in the hepatic trauma setting, especially in severe injuries. Hence, clinicians should be familiar with the concept of hepatic ischemia-reperfusion injury and respond appropriately and timely.
\end{abstract}

Keywords: Reperfusion Injury; Ischemia; Pathophysiology; Prevention

\section{Context}

Ischemia reperfusion injury (IRI) is defined as the phenomenon during which cellular damage in an organ, caused by hypoxia, is paradoxically exacerbated after the restoration of oxygen delivery (1). It is a dynamic process which involves the two interrelated phases of local ischemic insult and inflammation-mediated reperfusion injury (2). This concept occurs in several organ systems such as the central nervous system, liver, heart, lung, intestine, skeletal muscle, and kidney (3). If severe enough, the inflammatory response after IRI may even result in the systemic inflammatory response syndrome (SIRS) or the multiple organ dysfunction syndrome (MODS) (4). Hepatic IRI is a frequent and major complication in clinical practice, which compromises liver function and increases postoperative morbidity, mortality, recovery, and overall outcome. Liver, being an organ with high energy requirements, is highly dependent on oxygen supply and susceptible to hypoxic or anoxic conditions (5). Hepatic IRI can be categorized into warm and cold ischemia. Warm ischemia occurs in the setting of transplan- tation, trauma, shock, and elective liver surgery, in which hepatic blood supply is temporarily interrupted. It may also occur in some types of toxic liver injury, sinusoidal obstruction and Budd-Chiari syndrome (6). Cold storage ischemia occurs during organ preservation before transplantation. Numerous factors contribute to hepatic IRI, including Kupffer cells (KC) activation, oxidative stress and upregulation of proinflammatory cytokine signaling (7). This variety of mechanisms, contribute to various extents to the overall pathophysiology. Hence, it is difficult to achieve effective protection by targeting individual mediators or mechanisms and plentiful strategies reducing IRI, both clinical and experimental, have been extensively studied (8). Unfortunately, the outcomes of promising experimental studies cannot be always applied in the clinical setting. As far as the importance of hepatic IRI in trauma is concerned, despite the fact that the management of hepatic trauma has become increasingly nonoperative, there still remains the possibility of hepatic resection in the hepatic trauma setting, especially in severe injuries (9). Intraoperative cessation of he-

Implication for health policy/practice/research/medical education:

Hepatic ischemia and reperfusion injury is a complex phenomenon which is commonplace in clinical practice such as the operative management of hepatic trauma, liver transplantation and liver resection. Hence, surgeons should be familiar with it and apply the necessary methods to prevent it.

Copyright (C) 2013, Kashan University of Medical Sciences; License Kowsar Ltd. This is an Open Access article distributed under the terms of the Creative Commons Attribution License (http://creativecommons.org/licenses/by/3.0), which permits unrestricted use, distribution, and reproduction in any medium, provided the original work is properly cited. 
patic blood supply, by a variety of clamping maneuvers, is sometimes necessary during resection and inevitably exposes the liver to warm IRI. Furthermore, the liver with its role as the biochemical factory of sorts for the organism, as well as its anatomic and physiologic position, is vulnerable to the ischemia which is frequently encountered in patients with trauma. In this paper we would review the latest knowledge regarding the pathophysiology of hepatic ischemia reperfusion injury and present current and future options, both surgical and pharmacological, to attenuate it.

\section{Evidence Acquisition}

A review of the most recent literature was conducted using PubMed asa search engine with the focus on papers dealing with ischemia/reperfusion of the liver, especially relating to hepatic trauma or similar situations. Additionally, emphasis was given on identifying the most recent data on the mechanisms involved in hepatic ischemia/reperfusion injury.

\section{Results}

\subsection{Current Knowledge of the Pathophysiology of Hepatic I/R Injury}

During an ischemic period, several functional changes occur at the cellular level that promote cell injury (10). More specifically, a decrease in oxidative phosphorylation, results in Adenosine-5'-triphosphate (ATP) depletion and derangements in calcium homeostasis (11). The lack of oxygen to hepatocytes during ischemia also causes mitochondrial deenergization, alterations of $\mathrm{H}^{+}$ and $\mathrm{Na}^{+}$homeostasis, and finally swelling of the sinusoidal endothelial cells (SEC), and the KC (12). Activation of $\mathrm{KC}$ with production of reactive oxygen species (ROS), upregulation of the inducible nitric oxide synthase (iNOS) in hepatocytes, and upregulation of proinflammatory cytokines, chemokines, and adhesion molecules resulting in neutrophil-mediated injury, are all major contributing events to the inflammation-associated damage (13). Below are the hepatic IRI mechanisms and mediators.

\subsubsection{ATP Depletion}

Failure of aerobic ATP formation by oxidative phosphorylation is the fundamental stress of anoxic and ischemic injury. The importance of ATP depletion, in the events leading to necrotic cell death, is demonstrated by the ability of glycolytic substrates to rescue hepatocytes and sinusoidal endothelial cells from lethal cell injury (14). But glucose does not protect hepatocytes against anoxic injury since hepatocytes lack hexokinase. Endogenous glycogen is also an excellent substrate for anaerobic glycolysis (15). Fructose also prevents hepatocellular killing by several toxic chemicals, which implies that mitochondria are important targets of toxic cell killing (16). In ad- dition, during anoxia, mitochondrial respiration, and hence oxidative phosphorylation become fully inhibited.

\subsubsection{ROS Creation, Kupffer Cells, and Chemokine Secre- tion}

Reoxygenation of the hypoxic liver promotes the formation of ROS, including hydrogen peroxide $\left(\mathrm{H}_{2} \mathrm{O}_{2}\right)$ and superoxide $\left(\mathrm{O}^{2}-\right)$. The source of ROS in hepatic IRI has long been controversial. Regarding the mechanisms responsible for ROS production, experiments with xanthine oxidase (XOD)/xanthine dehydrogenase (XDH) inhibitors, such as allopurinol, suggest that the $\mathrm{XOD} / \mathrm{XDH}$ system is the main ROS generator in hepatocytes (10). However, other results obtained in experimental models of the isolated perfused liver have suggested that the mitochondria, through the respiratory chain, could be the main source of ROS (17). Oxidative stress can damage cells through multiple mechanisms, including lipid peroxidation, DNA oxidation, and enzyme denaturation. It is also known that ROS do not cause cytotoxicity directly, but act as signaling molecules that upregulate nuclear transcription factors like Nuclear Factor kB (NF-kB) and subsequently release Tumor Necrosis Factor-a (TNF- $\alpha$ ) and interleukin 1 (IL-1) (18). Additionally, ROS can cause damage from oxidant stress, which occurs during the early phase of injury, and activate inflammatory pathways that lead to neutrophil accumulation in the liver in the later phase. KCs are the resident macrophages of the liver and are critical in the earliest stages of IRI (19). They are responsible for the production of cytokines and chemokines which play a key role in the pathogenesis of IRI locally and systemically. However, the elimination of KC did not modify the deleterious effects of IRI and the activation of neutrophils is not essential for reoxygenation injury (20). TNF-a and IL-1 are the earliest cytokines to be increased in IRI, with increased levels occurring within minutes of reperfusion of the liver (21). Parenchymal cells of the liver may regulate the release of these cytokines during ischemic stress. Hepatocyte expression of interleukin-12 (IL-12) has been also shown to be a key element in the production of TNFa and IL-1 at reperfusion (22). The release of these molecules, which occurs from multiple cell types (KC, endothelial cells, hepatic parenchymal cells) finally results in a proinflammatory state caused by the resultant infiltration of immune cells in the injured organ (23). TNF- $\alpha$ is reported to have different effects in the IRI. It enhances oxidative stress-induced injury and induces apoptosis in hepatocytes, provided that protein synthesis or NF-kBmediated gene expression is suppressed (24). At the same time, studies have linked disruption of TNF- $\alpha$ release to decreased hepatic injury and increased liver regeneration (25). Other mediators taking part in the injury process are interleukin 6 (IL-6) and glutathione levels. IL-6 release is delayed, and IL- 6 treatment protects against warm IRI in rats (26). Decreased glutathione levels and elevated oxidized glutathione levels are used to demon- 
strate oxidative stress injury (27).

Additionally, IL-1 and TNF- $\alpha$ recruit and activate Cluster of Differentiation $4\left(\mathrm{CD}^{+}\right) \mathrm{T}$-lymphocytes, which produce granulocyte-macrophage colony-stimulating factor (GM-CSF), tumor necrosis factor $\beta$ and $\gamma$ (TNF- $\beta$ and TNF- $\gamma$ ). These cytokines amplify KC activation and TNF- $\alpha$ and IL-1 secretion and promote neutrophil recruitment and adherence into the liver sinusoids, thus creating somewhat of a circular pattern in the activation process (11).

\subsubsection{Neutrophil Accumulation}

Although chemokines serve to attract neutrophils to ischemic areas of injury via a chemokine gradient within the liver tissue, adhesion molecules such as intercellular adhesion molecule 1 (ICAM-1) with integrins such as CD11b/CD18 (also called Macrophage-1 antigen or MAC-1) are required for neutrophils to transmigrate from the bloodstream to the sites of injury $(28,29)$. Activation of neutrophils has been also implicated in the hepatic microvascular dysfunction and parenchymal damage associated with IRI (30). More specifically, the extensive vascular injury during reperfusion eliminates, in part, the sinusoidal endothelial cell barrier and the neutrophil has direct access to hepatocytes (31). When neutrophils infiltrate the liver, they worsen hepatic hypoperfusion, exacerbate the effects of Endothelin-1, and may release proteases through granule exocytosis (cathepsin G, granulocytes elastase) which are toxic to hepatocytes (31). In general, they perpetuate and amplify the injury by releasing many of the same mediators as KC, but often in much larger quantities.

\subsubsection{The Role of Nitric Oxide in IRI}

The involvement of nitric oxide (NO) in IRI cannot be easily distinguished between beneficial or harmful. Some authors have found that NO exerts a beneficial effect on IRI in different organs, tissues and cells; whereas, other studies report no effect or even a deleterious action of NO (32). NO is formed by the enzyme iNOS from the precursor aminoacid arginine in the bloodstream. The endothelial form of NOS (eNOS) is constitutively expressed in endothelial cells of the liver and the iNOS form is induced during IRI and regulated by many of the same cytokines (TNFa and IL-1 among others) which are involved in IRI $(33,34)$. The production of NO during IRI seems to disturb the microcirculation of the liver and leads to mitochondrial dysfunction. Moreover, NO may promote apoptosis by inducing cytochrome $\mathrm{c}(\mathrm{Cyt}-\mathrm{C})$ release and caspase activation, while it has been also shown to upregulate the antiapoptotic protein B-cell lymphoma 2 (Bcl-2) $(35,36)$.

\subsubsection{Apoptosis and Necrosis}

During reperfusion, TNF-a and other mediators activate many of the proteins involved in apoptosis, such as the proteases caspase-3 and caspase-8, along with mitochondria Cyt-C release to the cytoplasm (37). This sequence of events leads to DNA destruction and apoptosis (38). The activation of caspases has also been used to demonstrate apoptosis in rat SECs following cold IRI (39). On the other hand, newer studies oppose the view that most cells undergo apoptosis in response to either warm or cold IRI, believing that necrosis is the principle form of cell death (12). The events that occur during the anoxia and the different phases of the reperfusion injury are being summarized in Table 1.

\begin{tabular}{l}
\hline Table 1. Key Events in the Pathophysiology of Hepatic IRI \\
\hline Features \\
\hline Decrease in oxidative phosphorylation results in ATP deple- \\
tion \\
Derangement in $\mathrm{Na}^{+}, \mathrm{Ca}^{+2}{\mathrm{and} \mathrm{H}^{+} \text {homeostasis and mitochon- }}_{\text {drial deenergization }}$ \\
ROS $^{\mathrm{a}}$ formation \\
Upregulation of NFkB factor and TNF-a-IL-1 release \\
Cytokines and Chemokines production by Kupffer cells \\
Activation of CD4 ${ }^{+} \mathrm{T}$ helper cells \\
Neutrophil infiltration \\
NO ${ }^{\mathrm{a}}$ production \\
Hepatic cells apoptosis \\
\hline a Abbreviations: NO, nitric oxide; ROS, reactive oxygen species
\end{tabular}

\subsection{Contemporary Approaches to Prevent Hepatic IRI}

The predominant methods used to prevent the phenomenon of hepatic IRI are Ischemic Preconditioning (IP), in situ cooling of the liver and various pharmacological interventions. Furthermore, variations of existing liver clamping techniques, designed to reduce the IRI, have been extensively studied and are being routinely implemented during hepatic resections.

\subsubsection{Surgical Methods Used to Reduce Hepatic IRI}

During liver resection, it is usually necessary to apply various selective or nonselective vascular occlusion techniques to reduce blood loss, as the latter is associated with adverse postoperative outcomes. These include the continuous or intermittent Pringle Maneuver (PM), the total hepatic vascular exclusion (THVE), and the hemihepatic or segmental occlusion of the portal vein or hepatic artery (40). Sadly, the aforementioned methods are accompanied by negative effects such as the hepatic IRI (6). Hence, surgeons need to achieve the right balance between reduced blood loss and attenuation of IRI.

The PM is the simplest and the most widely used and time-honored method of liver vascular clamping. It involves the simultaneous clamping of the hepatic artery and portal vein. By using intermittent, instead of continuous PM, hepatic IRI can be reduced, as intermittent clamping appears to be better tolerated (41). The optimal 
ischemic intervals are still debated and numerous studies try to define the optimal PM cycle. All in all, ischemic intervals of 30 minutes can be safely used followed by 5 minutes of reperfusion (40). According to der Broek et al. there were no significant differences between ischemic intervals of 15 minutes and 30 minutes as far as hepatocellular injury, median blood loss, liver function and morbidity are concerned (42). THVE has the advantage of less blood loss during the operation, while enabling difficult vascular reconstructions or reimplantations. It does have several adverse effects such as hemodynamic intolerance, increased operative time, and longer postoperative hospital stay compared to the PM $(43,44)$. Nevertheless, the combination of in situ Hypothermic Perfusion (HP) of the remnant liver with the THVE is promising. HP, which is achieved with cytoprotective solutions in combination with local cooling of the organ's surface, has been used to prolong ischemic tolerance (45). Experimental studies have demonstrated that the parenchymal hypothermia reduces oxidative stress and the inflammatory response seen in IRI, thus allowing more complex hepatectomies to take place $(46,47)$.

\subsubsection{Ischemic Preconditioning}

Regarding the IP most evidence comes from animal experiments and clinical series showing contrasting views (48). IP is characterized by a short period of ischemia and reperfusion preceding a longer time of ischemia. Three studies, in 2000, 2003 and 2004 respectively, demonstrated that IP was associated with significant beneficial effects and reduction of hepatic IRI, both in patients with steatotic and with normal liver parenchyma (49-51). Furthermore, IP prior to the PM can improve liver macro-circulation because it increases arterial perfusion and prevents portal vein post-ischemic flow reduction (52). On the contrary, a series of recent studies suggest that there is no additional protective effect of IP in patients undergoing liver resection under continuous or intermittent vascular occlusion (PM, intermittent PM or THVE) (53, 54 ). The most recent one by Jeon et al. showed that postoperative liver function tests, the duration of the operation, and the hospital stay were not significantly different between THVE alone and THVE preceded by IP. The morbidity rates were $37.5 \%$ for total vascular exclusion alone (THVE), and $34.2 \%$ for THVE and IP, respectively. Moreover, the application of IP is not recommended in older patients $(55,56)$. However, using IP prior to intermittent PM is associated with reduced blood loss and shorter transection time (53).

\subsubsection{Pharmacological Approaches}

Hepatic IRI has a complex pathophysiologic background. A lot of cell types, inflammatory mediators and reactive oxygen mediators play an important role. As a result, a lot of pharmacological interventions are being tested to reduce the phenomenon of IRI.
Methylprednisolone, trimetazidine, glucose and ulinastatin may have protective roles against IRI in liver resection (57). Still, they remain controversial and cannot be routinely used to reduce IRI during controlled liver resections due to lack of clinical trials.

\subsubsection{Prednisolone}

Prednisolone a glucocorticoid steroid, acts as an antiinflammatory agent, reducing inflammatory markers and apoptotic cell count in experimental liver IRI (58). It decreased hospital stay and blood transfusion requirements, and improved liver function and showed a trend favoring a decreased postoperative complication rate (58). According to a 2013 systematic review and meta-analysis of the effect of perioperative steroids on IRI, perioperative steroids have a favorable impact on postoperative outcomes after liver resection. Patients receiving intravenous glucocorticoids were $24 \%$ less likely to have postoperative morbidity compared with controls. In addition, steroids significantly reduced postoperative blood levels of bilirubin and of inflammatory markers such as interleukin 6 (IL-6) and C-reactive protein (CRP). There was no evidence of a difference in infectious and wound healing complications between the study groups (59).

\subsubsection{Other Promising Pharmacological Interventions}

Glucose, when given in high-concentration intravenously during 24 hrs before the operation, has proved to be beneficial in alleviating liver IRI during hepatic vascular occlusion (60). According to this study, hepatic tissue ATP content in the experimental group was significantly higher at the end of both the hepatic vascular occlusion and the point of the one hour reperfusion. Furthermore, the liver function of the experimental group was significantly better than that of the control group the first and fifth day after the operation. As far as ulinastatin is concerned, although it lowered the transaminase and bilirubin levels, it did not affect the rates of liver decompensation, perioperative morbidity or length of hospital stay $(57,61)$. Based on these results, it seems that ulinastatin may offer a protective role in elective liver resections under vascular occlusion. Another promising pharmacological substance is the Urinary Trypsin Inhibitor (UTI). UTI acts by reducing NF-kB activation and thus attenuates hepatic IRI. Wu YJ et al. demonstrated the potential effects of UTI administration before and after the liver ischemia (62). When UTI was administered preoperatively, lower Alanine transaminase and Aspartate transaminase levels were observed, as well as reduced NF-kB activation. The pathological hepatocellular damage was also improved, as neutrophil aggregation and infiltration, which lead to hepatic IRI, were inhibited (62). Last but not least, trimetazidine, an antianginal drug, may have a role in protecting the liver during resection under vascular occlusion. It has been shown to decrease liver $\mathrm{I} / \mathrm{R}$ injury in experimental models by increasing ATP production and 
reducing oxygen consumption (58). Settaf et al. suggested that trimetazidine attenuates ischaemia-reperfusion injury during liver surgery (63). They demonstrated that the daily administration of trimetazidine five days prior to liver surgery, reduced cytolysis and increased liver ATP content. In addition, postoperative Aspartate transaminase (AST) and Alanine transaminase (ALT) levels and hospital stay were significantly lower in the trimetazidine group compared to the placebo group (63).

\subsection{Potential Future Therapeutic Approaches to Prevent Hepatic IRI}

The fact that hepatic cell damage, caused by IRI, can lead to severe complications, during or after liver transplantation or liver resections for hepatic trauma management, highlights the great need for research into this field, to gain more experience regarding the prevention of this undesirable effect.

The mechanisms responsible for hepatic IRI have been studied thoroughly and many attempts have been made to discover possible ways of successfully helping patients recover from major liver surgery. However, the fact that scientific research relies on the use of cell cultures and animal models, sets limitations on improving our knowledge on this subject, as most human tissues are not routinely accessible for research purposes. Despite the aforementioned difficulties, several methods about protecting the liver from IRI have been developed and tested in animal experimental models and significant efforts have been made to apply the results of these studies into clinical practice (1).

\subsubsection{Antioxidants}

The possibility of ROS and oxidative stress being responsible for the cell damage occurring during IRI, has led to a large volume of recent research around antioxidant therapy. While the results in animal models are promising, there is a need for similar successes in human trials, so that these therapies can proceed to the clinical level.

\subsubsection{Endogenous Genes and Gene Products}

Several gene products have proven to be protective when administered in the case of IRI. Generally, they tend to exert their effect mainly through the ROS (64). In this review, the use of glutathione and superoxide dismutase (SOD) would be discussed.

The tripeptide glutathione (GSH) is an antioxidant present in high concentrations in hepatocytes. Its levels are regulated by the Nuclear factor (erythroid-derived 2)-like 2 (Nrf 2) dependent gene glutaminate-cysteine ligase. It has been proven that intravenous administration of glutathione can effectively protect liver cells against reperfusion injury by detoxifying ROS (65). Studies have also demonstrated that treatment with the cysteine derivative, N-acetylcysteine (NAC), before or during IRI can maintain GSH levels and limit ROS. Furthermore, the fact that glutathione's half life is short in humans has led to the thought that gene transfer would be able to increase intracellular glutathione levels without the need of intravenous administration in the future (64).

Another antioxidant enzyme that has been studied for its protective role against IRI, is SOD. Because of SOD's poor bioavailability, the protection it offered, when administered intravenously, was not significant. On the other hand, when conjugated to carbohydrate factors, it proved to be much more effective. Gene transfer is an attractive option in this case too, but still at an early research stage (66).

\subsubsection{Mitochondrial Permeability Transition (MPT) inhi- bition:}

The MPT plays an important role during hepatic damage by IRI, as it escalates the oxidative stress. Therefore, inhibitors of the MPT have proven to effectively protect hepatic cells. One such inhibitor is Edaravone, which is considered to reduce hepatic injury in the early stage of IRI, as shown in a porcine hepatectomy model (67).

\subsubsection{Pharmacological Preconditioning}

As mentioned before, preconditioning is a procedure which involves exposing the liver to a brief period of ischemia and then reperfusion, before the actual period of hepatic ischemia. An alternative to the surgical preconditioning has been the effort to achieve pharmacologically preconditioning. Studies have shown that activating the Adenosine $2 \mathrm{~A}(\mathrm{~A} 2 \mathrm{~A})$ receptor using an A2A agonist, before the IRI, can protect against cell damage, as it mimics the physiological stimulation of the receptor that happens during IRI (68).

\subsubsection{Nitric Oxide}

A substantial body of literature testifies to the fact that NO is an important endogenous molecule, involved in IRI. First of all, NO eliminates the decreased ATP levels related to liver (69) IRI. It also prevents the elevation of cytokine levels, such as TNF-a, interleukin $\beta$ (IL- $\beta$ ) and IL-12, thereby decreasing the inflammatory process during which liver cell death occurs (70). As mentioned before, glutathione is associated with the protection of hepatic tissue during IRI, while NO mitigates oxidative damage by preventing reduction of this antioxidant (71). Last but not least, studies have shown that NO plays an important role in preserving the blood flow to the liver microcirculation during reperfusion, following a period of liver ischemia (69, 71). All these promising results have led to the hypothesis that exogenous NO could protect the liver. However, NO is a very unstable free radical and that is why its delivery needs another molecule to which NO would be attached to reach the liver. Some of the potential NO donors that could be used are S-nitrosothiols, diazeniumdiolates and liver-selective NO donors (72-76). All these molecules have 
been tested in numerous studies, with the S-nitrosothiols being the most studied of all.

\subsubsection{Nuclear Factor-kB (NF-kB)}

$\mathrm{NF}-\mathrm{kB}$ is an inducible nuclear transcription factor which regulates the expression of many genes and is also activated by a number of extracellular agents. NF-kB plays diverse roles in the acute injury response in both warm and cold hepatic ischemia. Activation of NF-kB in KC promotes inflammation through cytokine expression while activation in hepatocytes may be cell protective (77). With the multidimensional functions of NF-kB in reperfusioninjury, it should not come as a surprise that it has been implicated in numerous studies considering potential immunomodulatory therapeutic strategies. One of these studies has tried to determine whether the receptor activator of NF-kB (Receptor Activator of Nuclear Factor $\kappa B-R A N K)$ and its ligand (RANKL) are important in the hepatic response to IRI, as it is well known that their interaction promotes NF-kB activation (78). The study proved that treatment with RANKL, before ischemia or at reperfusion, increased hepatocyte NF-kB activation, leading to a significant reduction in liver cell damage.

\begin{tabular}{l}
\hline Table 2. Current and Potential Options to Prevent Hepatic IRI \\
\hline Current Surgical Options \\
\hline Intermittent pringle maneuver \\
\hline Hypothermic perfusion \\
Ischemic preconditioning \\
Current Pharmacological Options \\
Prednisolone \\
\hline Glucose \\
\hline Ulinastatin \\
\hline Urinary trypsin inhibitor \\
Trimetazidine \\
\hline Future Pharmacological Options \\
\hline Glutatheione \\
\hline Superoxide dismutases \\
\hline Inhibitors of mitochondrial permeability transition \\
\hline NO \\
\hline NF-kB \\
\hline Nilotinib \\
\hline
\end{tabular}

${ }^{\mathrm{a}}$ Abbreviations: NO, nitric oxide; NF-kB, nuclear factor $\mathrm{kB}$

\subsubsection{Nilotinib}

Even though antioxidants are the most studied solutions for preventing or reducing hepatic cell damage by IRI, there are other potential therapeutics. One of them is Nilotinib (receptor tyrosine kinase inhibitor), and its protective role against liver cell damage. One recent study has shown that Nilotinib lowers both liver Jun $\mathrm{N}$ terminal kinases (JNK) activation and neural progenitor cells (NPC) p 38 mitogen-activated protein kinase (MAPK) activation in mice, and may be useful in ameliorating liver IRI in humans (79). All of the aforementioned methods are summarized in Table 2.

\section{Conclusions}

Hepatic IRI is an intriguing subject which still surprises with the countless mediators and interactions between them. It has an adverse effect on morbidity and mortality rates following liver resection and consequently should be seriously considered when dealing with hepatic trauma. The intermittent PM and the IP, along with the perioperative use of steroids and the antioxidant therapies, are some of the current options in our effort to reduce IRI. However, there has been extensive research in the field of noninvasive liver protection from IRI, mainly in animal models. Hopefully, this research would prove effective at introducing new methods of protection against IRI in the clinical practice.

\section{Acknowledgements}

We would like to thank the medical library of Papageorgiou General Hospital for providing us access to relative medical books and journals.

\section{Financial Disclosure}

None of the authors had any financial interests related to the manuscript.

\section{Funding/Support}

There was no sponsor.

\section{Authors' Contribution}

Georgios Tsoulfas was responsible for the concept and design of the study and also for the critical revision of the manuscript. Dimitris Papadopoulos, Thomas Siempis and Eleni Theodorakou were responsible for the research and drafting the manuscript.

\section{References}

1. Elias-Miró M, Jiménez Castro M, Peralta C. Ischemia-Reperfusion Injury Associated with Liver Transplantation in 2011: Past and Future. 2012.

2. Zhai Y, Petrowsky H, Hong JC, Busuttil RW, Kupiec-Weglinski JW. Ischaemia-reperfusion injury in liver transplantation-from bench to bedside. Nat Rev Gastroenterol Hepatol. 2013;10(2):79-89.

3. Eltzschig HK, Collard CD. Vascular ischaemia and reperfusion in jury. Br Med Bull. 2004;70:71-86.

4. Videla LA. Oxidative stress signaling underlying liver disease and hepatoprotective mechanisms. World J Hepatol. 2009;1(1):72-8.

5. Teoh NC. Hepatic ischemia reperfusion injury: Contemporary perspectives on pathogenic mechanisms and basis for hepa- 
toprotection-the good, bad and deadly. J Gastroenterol Hepatol. 2011;26 Suppl 1:180-7.

6. Fernandez V, Tapia G, Videla LA. Recent advances in liver preconditioning: Thyroid hormone, n-3 long-chain polyunsaturated fatty acids and iron. World J Hepatol. 2012;4(4):119-28.

7. van Golen RF, Reiniers MJ, Olthof PB, van Gulik TM, Heger M. Sterile inflammation in hepatic ischemia/reperfusion injury: present concepts and potential therapeutics. J Gastroenterol Hepatol. 2013;28(3):394-400.

8. Swift C, Garner JP. Non-operative management of liver trauma.J RArmy Med Corps. 2012;158(2):85-95.

9. Banga NR, Homer-Vanniasinkam S, Graham A, Al-Mukhtar A, White SA, Prasad KR. Ischaemic preconditioning in transplantation and major resection of the liver. BrJ Surg. 2005;92(5):528-38.

10. Casillas-Ramirez A, Mosbah IB, Ramalho F, Rosello-Catafau J, Peralta $\mathrm{C}$. Past and future approaches to ischemia-reperfusion lesion associated with liver transplantation. Life Sci. 2006;79(20):1881-94.

11. De Groot H, Rauen U. Ischemia-reperfusion injury: processes in pathogenetic networks: a review. Transplant Proc. 2007;39(2):481-4.

12. Massip-Salcedo M, Rosello-Catafau J, Prieto J, Avila MA, Peralta C. The response of the hepatocyte to ischemia. Liver Int. 2007;27(1):6-16.

13. Bilzer M, Gerbes AL. Preservation injury of the liver: mechanisms and novel therapeutic strategies. J Hepatol. 2000;32(3):508-15.

14. Nishimura Y, Romer LH, Lemasters JJ. Mitochondrial dysfunction and cytoskeletal disruption during chemical hypoxia to cultured rat hepatic sinusoidal endothelial cells: the $\mathrm{pH}$ paradox and cytoprotection by glucose, acidotic $\mathrm{pH}$, and glycine. Hepatology. 1998;27(4):1039-49.

15. Bradford BU, Marotto M, Lemasters JJ, Thurman RG. New, simple models to evaluate zone-specific damage due to hypoxia in the perfused rat liver: time course and effect of nutritional state. $J$ Pharmacol Exp Ther. 1986;236(1):263-8.

16. Nieminen AL, Dawson TL, Gores GJ, Kawanishi T, Herman B, Lemasters JJ. Protection by acidotic $\mathrm{pH}$ and fructose against lethal injury to rat hepatocytes from mitochondrial inhibitors, ionophores and oxidant chemicals. Biochem Biophys Res Commun. 1990;167(2):600-6.

17. Jaeschke H, Mitchell JR. Mitochondria and xanthine oxidase both generate reactive oxygen species in isolated perfused rat liver after hypoxic injury. Biochem Biophys Res Commun. 1989;160(1):140-7.

18. Cursio R, Gugenheim J, Ricci JE, Crenesse D, Rostagno P, Maulon $\mathrm{L}$, et al. A caspase inhibitor fully protects rats against lethal normothermic liver ischemia by inhibition of liver apoptosis. FASEB J.1999;13(2):253-61.

19. Jaeschke H, Smith CV, Mitchell JR. Reactive oxygen species during ischemia-reflow injury in isolated perfused rat liver. J Clin Invest. 1988;81(4):1240-6.

20. Imamura H, Sutto F, Brault A, Huet PM. Role of Kupffer cells in cold ischemia/reperfusion injury of rat liver. Gastroenterology. 1995;109(1):189-97.

21. Wanner GA, Ertel W, Muller P, Hofer Y, Leiderer R, Menger MD, et al. Liver ischemia and reperfusion induces a systemic inflammatory response through Kupffer cell activation. Shock. 1996;5(1):34-40.

22. Lentsch AB, Yoshidome H, Kato A, Warner RL, Cheadle WG, Ward $\mathrm{PA}$, et al. Requirement for interleukin-12 in the pathogenesis of warm hepatic ischemia/reperfusion injury in mice. Hepatology. 1999;30(6):1448-53.

23. Colletti LM, Kunkel SL, Walz A, Burdick MD, Kunkel RG, Wilke $\mathrm{CA}$, et al. The role of cytokine networks in the local liver injury following hepatic ischemia/reperfusion in the rat. Hepatology. 1996;23(3):506-14.

24. Bradham CA, Qian T, Streetz K, Trautwein C, Brenner DA, Lemasters JJ. The mitochondrial permeability transition is required for tumor necrosis factor alpha-mediated apoptosis and cytochrome c release. Mol Cell Biol. 1998;18(11):6353-64.

25. Tian Y, Jochum W, Georgiev P, Moritz W, Graf R, Clavien PA. Kupffer cell-dependent TNF-alpha signaling mediates injury in the arterialized small-for-size liver transplantation in the mouse. Proc Natl Acad Sci U S A. 2006;103(12):4598-603.

26. Camargo CA, Jr, Madden JF, Gao W, Selvan RS, Clavien PA. Interleukin-6 protects liver against warm ischemia/reperfusion injury and promotes hepatocyte proliferation in the rodent. Hepatology. 1997;26(6):1513-20.

27. Ghosh S, May MJ, Kopp EB. NF-kappa B and Rel proteins: evolutionarily conserved mediators of immune responses. Annu Rev Immunol. 1998;16:225-60.

28. Burke J, Zibari GB, Brown MF, Granger N, Kelly R, Singh I, et al. Hepatic ischemia-reperfusion injury causes E-selectin upregulation. Transplant Proc. 1998;30(5):2321-3.

29. Lentsch AB, Yoshidome H, Cheadle WG, Miller FN, Edwards MJ. Chemokine involvement in hepatic ischemia/reperfusion injury in mice: roles for macrophage inflammatory protein-2 and KC. Hepatology. 1998;27(4):1172-7.

30. Cutrn JC, Perrelli MG, Cavalieri B, Peralta C, Rosell Catafau J, Poli G. Microvascular dysfunction induced by reperfusion injury and protective effect of ischemic preconditioning. Free Radic Biol Med. 2002;33(9):1200-8.

31. McKeown CM, Edwards V, Phillips MJ, Harvey PR, Petrunka CN, Strasberg SM. Sinusoidal lining cell damage: the critical injury in cold preservation of liver allografts in the rat. Transplantation. 1988;46(2):178-91.

32. Peralta C, Rull R, Rimola A, Deulofeu R, Rosello-Catafau J, Gelpi E, et al. Endogenous nitric oxide and exogenous nitric oxide supplementation in hepatic ischemia-reperfusion injury in the rat. Transplantation. 2001;71(4):529-36.

33. Geller DA, Nussler AK, Di Silvio M, Lowenstein CJ, Shapiro RA, Wang SC, et al. Cytokines, endotoxin, and glucocorticoids regulate the expression of inducible nitric oxide synthase in hepatocytes. Proc Natl Acad Sci US A. 1993;90(2):522-6.

34. Hur GM, Ryu YS, Yun HY, Jeon BH, Kim YM, Seok JH, et al. Hepatic ischemia/reperfusion in rats induces iNOS gene transcription by activation of NF-kappaB. Biochem Biophys Res Commun. 1999;261(3):917-22.

35. Chung HT, Pae HO, Choi BM, Billiar TR, Kim YM. Nitric oxide as a bioregulator of apoptosis. Biochem Biophys Res Commun. 2001;282(5):1075-9.

36. Genaro AM, Hortelano S, Alvarez A, Martinez C, Bosca L. Splenic B lymphocyte programmed cell death is prevented by nitric oxide release through mechanisms involving sustained Bcl-2 levels. J Clin Invest. 1995;95(4):1884-90.

37. Arumugam TV, Shiels IA, Woodruff TM, Granger DN, Taylor SM. The role of the complement system in ischemia-reperfusion injury. Shock. 2004;21(5):401-9.

38. Gao W, Bentley RC, Madden JF, Clavien PA. Apoptosis of sinusoidal endothelial cells is a critical mechanism of preservation injury in rat liver transplantation. Hepatology. 1998;27(6):1652-60.

39. Natori S, Selzner M, Valentino KL, Fritz LC, Srinivasan A, Clavien PA, et al. Apoptosis of sinusoidal endothelial cells occurs during liver preservation injury by a caspase-dependent mechanism. Transplantation. 1999;68(1):89-96.

40. Hoekstra LT, van Trigt ID, Reiniers MJ, Busch OR, Gouma DJ, van Gulik TM. Vascular occlusion or not during liver resection: the continuing story. Dig Surg. 2012;29(1):35-42.

41. Lesurtel M, Lehmann K, de Rougemont O, Clavien PA. Clamping techniques and protecting strategies in liver surgery. $\mathrm{HPB}(\mathrm{OX}$ ford). 2009;11(4):290-5.

42. van den Broek MA, Bloemen JG, Dello SA, van de Poll MC, Olde Damink SW, Dejong CH. Randomized controlled trial analyzing the effect of 15 or 30 min intermittent Pringle maneuver on hepatocellular damage during liver surgery. $J$ Hepatol. 2011;55(2):337-45.

43. Belghiti J, Noun R, Zante E, Ballet T, Sauvanet A. Portal triad clamping or hepatic vascular exclusion for major liver resection. A controlled study. Ann Surg. 1996;224(2):155-61.

44. van Gulik TM, de Graaf W, Dinant S, Busch OR, Gouma DJ. Vascular occlusion techniques during liver resection. Dig Surg. 2007;24(4):274-81.

45. Hoti E, Salloum C, Azoulay D. Hepatic resection with in situ hypothermic perfusion is superior to other resection techniques. Dig Surg. 2011;28(2):94-9.

46. Azoulay D, Eshkenazy R, Andreani P, Castaing D, Adam R, Ichai P, et al. In situ hypothermic perfusion of the liver versus standard total vascular exclusion for complex liver resection. Ann Surg. 
2005;241(2):277-85

47. Dinant S, Roseboom HJ, Levi M, van Vliet AK, van Gulik TM. Hypothermic in situ perfusion of the porcine liver using Celsior or Ringer-lactate solution. Langenbecks Arch Surg. 2009;394(1):143-50.

48. Jeon J, Watkins A, Wagener G, Samstein B, Guarrera J, Goldstein $\mathrm{M}$, et al. Complex hepatectomy under total vascular exclusion of the liver: impact of ischemic preconditioning on clinical outcomes. World J Surg. 2013;37(4):838-46.

49. Chouker A, Schachtner T, Schauer R, Dugas M, Lohe F, Martignoni A, et al. Effects of Pringle manoeuvre and ischaemic preconditioning on haemodynamic stability in patients undergoing elective hepatectomy: a randomized trial. Br J Anaesth. 2004;93(2):204-11.

50. Clavien PA, Selzner M, Rudiger HA, Graf R, Kadry Z, Rousson V, et al. A prospective randomized study in 100 consecutive patients undergoing major liver resection with versus without ischemic preconditioning. Ann Surg. 2003;238(6):843-50.

51. Clavien PA, Yadav S, Sindram D, Bentley RC. Protective effects of ischemic preconditioning for liver resection performed under inflow occlusion in humans. Ann Surg. 2000;232(2):155-62.

52. Heizmann O, Meimarakis G, Volk A, Matz D, Oertli D, Schauer RJ Ischemic preconditioning-induced hyperperfusion correlates with hepatoprotection after liver resection. World J Gastroenterol. 2010;16(15):1871-8.

53. Petrowsky H, McCormack L, Trujillo M, Selzner M, Jochum W, Clavien PA. A prospective, randomized, controlled trial comparing intermittent portal triad clamping versus ischemic preconditioning with continuous clamping for major liver resection. Ann Surg. 2006;244(6):921-8.

54. Zapletal C, Fallsehr C, Reidel M, Loffler T, Gebhard MM, Golling M, et al. Induction of HSP70 shows differences in protection against I/R injury derived by ischemic preconditioning and intermittent clamping. Microvasc Res. 2010;80(3):365-71.

55. Bahde R, Spiegel HU. Hepatic ischaemia-reperfusion injury from bench to bedside. Br J Surg. 2010;97(10):1461-75.

56. Peters J, Nieuwenhuijs VB, Morphett A, Porte RJ, Padbury RT, Barritt GJ. Increasing cycles of intermittent ischemia can effectively maintain liver function during the acute phase of ischemia reperfusion injury by promotion of bile flow and reduction in bile salt toxicity. Dig Surg. 2009;26(6):455-64.

57. Abu-Amara M, Gurusamy K, Hori S, Glantzounis G, Fuller B, Davidson BR. Systematic review of randomized controlled trials of pharmacological interventions to reduce ischaemia-reperfusion injury in elective liver resection with vascular occlusion. HPB (Oxford). 2010;12(1):4-14.

58. Saidi RF, Chang J, Verb S, Brooks S, Nalbantoglu I, Adsay V, et al. The effect of methylprednisolone on warm ischemia-reperfusion injury in the liver. Am J Surg. 2007;193(3):345-7.

59. Orci LA, Toso C, Mentha G, Morel P, Majno PE. Systematic review and meta-analysis of the effect of perioperative steroids on ischaemia-reperfusion injury and surgical stress response in patients undergoing liver resection. Br J Surg. 2013;100(5):600-9.

60. Tang L, Tian F, Tao W, Cui J. Hepatocellular glycogen in alleviation of liver ischemia-reperfusion injury during partial hepatectomy. World J Surg. 2007;31(10):2039-43.

61. Li SQ, Liang LJ. Protection of Liver Function with Protease Inhibitor From Ischaemia-reperfusion Injury in Hepatocellular Carcinoma Patients Undergoing Hepatectomy after Hepatic Inflow Occlusion. Chin J Bases Clin Gen Surg. 2004;11:61-4.

62. Wu YJ, Ling Q, Zhou XH, Wang Y, Xie HY, Yu JR, et al. Urinary tryp- sin inhibitor attenuates hepatic ischemia-reperfusion injury by reducing nuclear factor-kappa B activation. Hepatobiliary Pancreat Dis Int. 2009;8(1):53-8.

63. Settaf A, Zaim N, Bellouch M, Tillement JP, Morin D. [Trimetazi dine prevents ischemia-reperfusion injury in hepatic surgery under vascular clamping]. Therapie. 2001;56(5):569-74.

64. Jaeschke H, Woolbright BL. Current strategies to minimize hepatic ischemia-reperfusion injury by targeting reactive oxygen species. Transplant Rev (Orlando). 2012;26(2):103-14.

65. Schauer RJ, Gerbes AL, Vonier D, Meissner H, Michl P, Leiderer R, et al. Glutathione protects the rat liver against reperfusion injury after prolonged warm ischemia. Ann Surg. 2004;239(2):220-31.

66. Yabe Y, Kobayashi N, Nishihashi T, Takahashi R, Nishikawa M, Takakura Y, et al. Prevention of neutrophil-mediated hepatic ischemia/reperfusion injury by superoxide dismutase and catalase derivatives. J Pharmacol Exp Ther. 2001;298(3):894-9.

67. Shimoda M, Iwasaki Y, Okada T, Kubota K. Edaravone inhibits apoptosis caused by ischemia/reperfusion injury in a porcine hepatectomy model. World J Gastroenterol. 2012;18(27):3520-6.

68. Arai M, Thurman RG, Lemasters JJ. Contribution of adenosine $\mathrm{A}(2)$ receptors and cyclic adenosine monophosphate to protective ischemic preconditioning of sinusoidal endothelial cells against Storage/Reperfusion injury in rat livers. Hepatology. 2000;32(2):297-302.

69. Koti RS, Yang W, Dashwood MR, Davidson BR, Seifalian AM. Effect of ischemic preconditioning on hepatic microcirculation and function in a rat model of ischemia reperfusion injury. Liver Transpl. 2002;8(12):1182-91.

70. Liu P, Xu B, Spokas E, Lai PS, Wong PY. Role of endogenous nitric oxide in TNF-alpha and IL-1beta generation in hepatic ischemiarepefusion. Shock. 2000;13(3):217-23.

71. Kobayashi H, Nonami T, Kurokawa T, Takeuchi Y, Harada A, Nakao A, et al. Role of endogenous nitric oxide in ischemia-reperfusion injury in rat liver. J Surg Res. 1995;59(6):772-9.

72. Aldemir M, Bosnak M, Al B, Buyukbayram H, Tacyildiz I. Effects of molsidomine and lexipafant in hepatic ischaemia--reperfusion injury. Injury. 2004;35(3):232-7.

73. Al-Sa'doni H, Ferro A. S-Nitrosothiols: a class of nitric oxide-donor drugs. Clin Sci (Lond). 2000;98(5):507-20.

74. DuMond JF, King SB. The chemistry of nitroxyl-releasing compounds. Antioxid Redox Signal. 2011;14(9):1637-48.

75. Katsumi H, Nishikawa M, Yasui H, Yamashita F, Hashida M. Prevention of ischemia/reperfusion injury by hepatic targeting of nitric oxide in mice. J Control Release. 2009;140(1):12-7.

76. Ricciardi R, Foley DP, Quarfordt SH, Saavedra JE, Keefer LK, Wheeler SM, et al. V-PYRRO/NO: an hepato-selective nitric oxide donor improves porcine liver hemodynamics and function after ischemia reperfusion. Transplantation. 2001;71(2):193-8.

77. Kuboki S, Okaya T, Schuster R, Blanchard J, Denenberg A, Wong $\mathrm{HR}$, et al. Hepatocyte NF-kappaB activation is hepatoprotective during ischemia-reperfusion injury and is augmented by ischemic hypothermia. Am J Physiol Gastrointest Liver Physiol. 2007;292(1):G201-7.

78. Sakai N, Van Sweringen HL, Schuster R, Blanchard J, Burns JM, Tevar $\mathrm{AD}$, et al. Receptor activator of nuclear factor-kappaB ligand (RANKL) protects against hepatic ischemia/reperfusion injury in mice. Hepatology. 2012;55(3):888-97.

79. Ocuin LM, Zeng S, Cavnar MJ, Sorenson EC, Bamboat ZM, Greer JB, et al. Nilotinib protects the murine liver from ischemia/reperfusion injury.J Hepatol. 2012;57(4):766-73. 